\title{
Habilidades directivas. Estrategias de formación turística en la Universidad de La Habana.
}

\section{Management skills. Strategies for tourism training at the University of Havana.}

MSc. Jimmy Eduardo Ascón Villa., ${ }^{1} \&$ Dra. Máryuri García González. ${ }^{2}$

\section{Resumen.}

Las habilidades directivas adquieren gran relevancia en las Instituciones de Educación Superior, y en el desempeño de sus cuadros dentro de los cuales los ejecutivos juegan un rol determinante. Las habilidades se encuentran vinculadas al liderazgo de sus directivos, y ponen de manifiesto sus características a través del desempeño. Es por ello que la presente se propone evaluar las habilidades directivas de los cuadros vinculados a la educación superior en turismo en la Universidad de La Habana. Con el fin de cumplir dicho objetivo, se tomaron en cuenta métodos empíricos y teóricos, que permitieron determinar en cuanto a importancia y desempeño el estado actual en el que se encuentran las habilidades. Se pudo establecer el nivel de desarrollo y el liderazgo transformacional como el estilo representativo a partir del que se proponen las estrategias.

Palabras claves: Habilidades directivas, estrategias, liderazgo, turismo.

\begin{abstract}
.
Management skills acquire great relevance in Higher Education Institutions, and in the performance of their cadres within which executives play a determining role. The skills are linked by the leadership of their managers, and show their characteristics through performance. That is why this is intended by assess the managerial skills of the cadres linked to higher education in tourism at the University of Havana. In order to meet this objective, empirical and theoretical methods were taken into account, which allowed determining in terms of importance and performance the current state in which the skills are located. The level of development and transformational leadership could be established as the representative style from which the strategies are proposed.
\end{abstract}

Keywords: Managerial skills, strategies, leadership, tourism.

\footnotetext{
${ }^{1}$ Universidad de La Habana, Facultad De Turismo. La Habana, Cuba. jascon@ ftur.uh.cu

${ }^{2}$ Universidad de La Habana Centro de Estudios para el Perfeccionamiento de la Educación Superior. La Habana, Cuba. maryuri@cepes.uh.cu
} 


\section{Introducción}

Las Instituciones de Educación Superior (en lo adelante IES) demandan que en el nuevo ámbito educativo se desempeñen por parte de los directivos las habilidades necesarias para mantener la calidad en su gestión.

Dichos retos ubican a los cuadros ejecutivos de dichas IES a replantearse las habilidades directivas básicas que son contempladas en la Metodología para el Proceso de Evaluación de los Cuadros del Estado y del Gobierno. (ENPYSC,2010)

La motivación y el compromiso con los objetivos educativos deben estar presente en este proceso e integrarse en las habilidades. Lo anterior obliga a determinar los niveles no solo de desempeño sino también de importancia de determinados tipos de conocimientos y habilidades que deben poseer los cuadros, incorporando a este proceso la formación de habilidades en los cuadros docentes, a través de un procedimiento de evaluación insertado en las estrategias de capacitación y superación de los cuadros y reservas.

La presente se encamina en la solución de la problemática anterior, que presenta una importancia práctica en el plano educacional determinada por una propuesta de estrategias para la gestión y mejora continua de las habilidades directivas para el desempeño de los cuadros ejecutivos. (Godet,2016)

El ejercicio del liderazgo por los directivos se justifica por el hecho de que, al hablar de centros educativos, tenemos una organización donde intervienen diferentes personas o grupos de éstas, con concepciones, visiones e intereses distintos. Por tanto, esto requiere alcanzar niveles significativos de acuerdos, de visión compartida y de sinergia que promuevan el logro de los fines y objetivos en las Instituciones de Educación Superior.

Problema: ¿Qué estrategias son necesarias para la formación de habilidades directivas en la educación superior en turismo en la Universidad de La Habana?

Hipótesis: El diseño de estrategias permitirá la formación de habilidades en los directivos de la enseñanza superior de turismo en la Universidad de La Habana.

Objetivo general: Elaborar estrategias para la formación de habilidades en los directivos de la educación superior en turismo de la Universidad de La Habana.

\section{Objetivos específicos:}

1. Caracterizar los fundamentos teóricos vinculados a la dirección estratégica de habilidades directivas.

2. Identificar las habilidades directivas que inciden en el liderazgo de los cuadros ejecutivos de la Facultad de Turismo de la Universidad de La Habana.

3. Evaluar el desempeño de las habilidades directivas de los cuadros ejecutivos de la Facultad de Turismo de la Universidad de La Habana.

La presente resulta vital para el departamento de Cuadro de la Universidad de La Habana y la Facultad de Turismo, así como garantiza un aporte teórico a la estrategia nacional de 
superación de cuadros y reservas al integrar un conjunto de técnicas comúnmente empleadas en análisis independientes, en aras de lograr un estudio holístico de las habilidades directivas de los cuadros ejecutivos vinculados a la enseñanza superior en turismo en dicha Institución de Educación Superior. Los resultados obtenidos pueden ser valorados en el resto de las universidades cubanas que se orientan a la formación de gestores del turismo. El estudio resulta novedoso para la IES siendo la primera vez que se desarrolla un análisis sobre las habilidades de sus directivos, y contribuye a la estrategia nacional para la superación y evaluación de dichos cuadros.

\section{Marco teórico}

\section{Las habilidades directivas y las estrategias de formación en función del liderazgo.}

Entre las esferas de las técnicas, a la cual los especialistas han ofrecido una atención especial en los últimos años, es la denominada habilidad directiva, que engloban principalmente las relaciones interpersonales que se desarrollan en el proceso de dirección de las IES, con: los subordinados, los niveles superiores, y los diferentes factores del entorno.

Según Codina (2016) es muy difícil que un dirigente logre dominar todos los aspectos técnicos y organizativos que se requieren para alcanzar los objetivos de su organización. Por tanto, su habilidad principal debe estar en lograr que su equipo y los miembros de su organización pongan conocimientos, experiencias y energías en función de los objetivos propuestos y de los resultados que se esperan de su gestión. Esta es la dirección principal a la que se dirige el desarrollo de las habilidades directivas.

Las habilidades directivas son el vehículo mediante el cual la estrategia y la práctica de la administración, las herramientas y las técnicas, los atributos de la personalidad y el estilo trabajan para producir resultados eficaces dentro de las organizaciones. En otras palabras, las habilidades directivas son los bloques de construcción sobre los que descansa la administración efectiva. (Whetten \& Cameron, 2011)

El perfeccionamiento de las habilidades directivas básicas para la gestión constituye un desafío en el acontecer de la educación superior. (Astigarraga, 2016)

Para un mejor entendimiento del término "habilidades directivas", Whetten y Cameron (2011) establecen cinco propiedades que las particularizan:

Primeramente, son conductuales; no son atributos de la personalidad o tendencias de estilo. Las habilidades directivas consisten en conjuntos identificables de acciones que los individuos llevan a cabo y que conducen a ciertos resultados. En segundo lugar, las habilidades administrativas son controlables. El desempeño de estos comportamientos se encuentra bajo control del individuo, el cual las puede demostrar, practicar, mejorar o limitar. En tercer lugar, pueden ser desarrolladas. Es posible mejorar el desempeño. A diferencia del cociente intelectual (ci) o de ciertos atributos de la personalidad o del temperamento, los cuales permanecen relativamente constantes a lo largo de la vida. En cuarto lugar, están interrelacionadas y se traslapan. Es difícil demostrar una sola 
habilidad aislada de las demás. Las habilidades no son comportamientos simplistas y repetitivos, sino conjuntos integrados de respuestas complejas. En quinto lugar, las habilidades directivas a veces son contradictorias y paradójicas. Por ejemplo, no todas las habilidades directivas fundamentales tienen una orientación suave y humanista, ni todas son impulsoras y marcan una dirección. No están orientadas exclusivamente hacia el trabajo en equipo o hacia las relaciones interpersonales. Los directivos más eficaces suelen poseer una variedad de habilidades, y algunas de ellas parecen incompatibles.

Iniciado el sigo XXI, el concepto de liderazgo se une de manera inseparable al cambio y a la transformación. La visión del liderazgo es un valor sustancial en las habilidades directivas de poder ejercer como motor del cambio, ya sea social, económico o de otro tipo. Ha surgido el líder multidisciplinar. Un liderazgo de múltiples vértices y de enormes capacidades creativas. (Bayón, 2015)

Las diferentes definiciones del término liderazgo, no tienen problemas en sí mismas, la mayoría dicen fundamentalmente lo mismo: el liderazgo tiene que ver con una persona que logra que otras hagan. Donde las definiciones difieren es en cómo los líderes motivan a sus seguidores, y en quién tiene voz y voto para diseñar los objetivos del grupo o de la organización. (Ronda,2015)

El término estrategia históricamente provine de la palabra griega stratégós (general) y en sus orígenes emerge como el arte de dirigir las operaciones militares. Luego, durante la segunda mitad del Siglo XX tuvo su extensión en la dimensión empresarial y académica. (Chamorro,2015)

El proceso para lograr esto puede resumirse en la definición de estrategia, que planteó Hatten (1987) a finales de los años ochenta sobre dirección estratégica como el proceso a través del cual una organización formula objetivos, y está dirigido a la obtención de los mismos. Estrategia es el medio, la vía, la directriz o camino creativo para la obtención de los objetivos de la organización. Es el arte de entremezclarse el análisis interno y la sabiduría utilizada por los dirigentes para crear valores, de los recursos y habilidades que ellos controlan.

En la actualidad podemos apreciar en los equipos y en las organizaciones aflora el estilo de liderazgo transformacional que se hace cada vez más característico de la IES como señala Gómez (2013) al identificar qué estos verifican lo que necesitan sus subordinados para cumplir los objetivos, aclaran funciones y tareas, instauran una estructura organizacional, premian el desempeño y toman en cuenta las necesidades sociales de sus seguidores. Trabajan intensamente e intentan dirigir a la organización con toda eficiencia y eficacia.

\section{Metodología}

La presente investigación es "descriptiva", porque se caracterizan las habilidades 
directivas como objeto de estudio. Además, se refleja un análisis "cuantitativo" y "cualitativo". De la misma manera, según la estrategia metodológica abordada, se puede clasificar como "investigación-acción", porque se parte de la realidad existente en los directivos y reservas para diagnosticar su situación.

\section{Fundamentación teórica de la investigación}

\section{Métodos teóricos:}

$\checkmark$ Histórico-Lógico: El método histórico permitió el estudio de la evolución de las habilidades directivas, sus tendencias actuales y la posibilidad de aplicación en la Facultad de Turismo de La Universidad de La Habana. Se analizaron investigaciones realizadas por importantes exponentes de la visión de futuro en el campo de las Instituciones de Educación Superior.

$\checkmark$ Hipotético-deductivo: El uso de este método facilitó la formulación de la hipótesis de la investigación como relación entre las variables habilidades directivas en los cuadros ejecutivos y sus estrategias de mejora en función del liderazgo futuro. El planteamiento de esta hipótesis y el desarrollo teórico de la investigación permitió arribar a conclusiones.

$\checkmark$ Análisis-síntesis: Posibilitó la búsqueda y el procesamiento de la información a partir de la bibliografía consultada y del estudio teórico de la temática investigada. Permitió determinar las características del estudio.

\section{Evaluación de las habilidades directivas en los cuadros ejecutivos de la Facultad de Turismo.}

La estrategia de preparación y superación es parte integrante del sistema de trabajo con los cuadros y sus reservas. Dentro de sus exigencias se encuentra:

$\checkmark$ El fortalecimiento de las habilidades directivas de los cuadros.

$\checkmark \quad$ La evaluación del impacto de la preparación y superación, valorando su efecto en el cuadro y sus reservas, a partir del resultado de su trabajo y el cumplimiento de las funciones del cargo que ocupa.

Dentro de los contenidos generales imprescindibles que se estructuran de acuerdo con la categorización de los cuadros ejecutivos se encuentra la preparación en habilidades directivas que abarca el conjunto de temáticas principales dirigidas a preparar a los cuadros y sus reservas acorde a las funciones de los cargos, para coadyuvar a elevar la eficiencia en su gestión. (Hernández, 2014)

\section{Técnicas Empleadas en el análisis de resultados}

Observación: Se aplicó con el propósito de analizar las habilidades.

\section{- Encuesta:}

Se aplicó para conocer el estado actual de las habilidades, según la percepción de su realización e importancia. Se empleó el método intencional encuestando a los diez 
cuadros ejecutivos de la Facultad de Turismo de la Universidad de La Habana.

\section{Resultados}

\section{Resultados de la encuesta aplicada a los cuadros ejecutivos de la Facultad de Turismo de la Universidad de La Habana}

La información obtenida a partir de las encuestas aplicadas a los directivos de la Facultad de Turismo de La Universidad de La Habana ofrece datos significativos en cuanto a la incidencia de aspectos instructivos en el desarrollo de las habilidades directivas.

El criterio de los encuestados en relación a los motivos que influyen negativamente en la mejora de habilidades directivas se expone en la figura 1. Dichos criterios evidencian que la falta de tiempo entre los cuadros para la formación de sus habilidades constituye el factor de mayor incidencia (96\%), seguido de la ausencia de programas de capacitación destinados al desarrollo de las mismas (78\%).

Figura N.1: Respuestas de los directivos para la variable "influencias negativas".

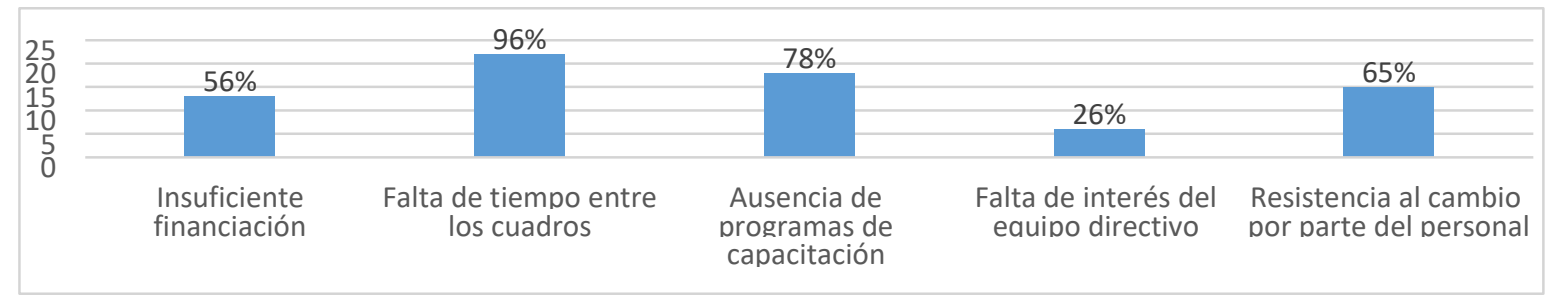

Fuente: Elaboración propia

Al evaluar las temáticas de mayor y menor incidencia en el desempeño de las habilidades directivas destacaron según la representación de la figura 2 como más influyentes la planificación estratégica, las herramientas de gestión prospectiva estratégica, la gestión del proceso enseñanza-aprendizaje, la gestión de personas y la responsabilidad social universitaria; así como otras de menor influencia como la comercialización, el diseño organizativo, el desarrollo de infraestructuras, el multiculturalismo y la gestión de la innovación. Al analizar la importancia atribuida por los cuadros encuestados a las actividades de formación de habilidades directivas, como muestra la Figura 2, se determinó que dentro de las más relevantes se encuentran los cursos de formación presencial (87\%), las consultorías (52\%), los entrenamientos y talleres (ambas 40\%) de frecuencia de selección; a la par que se revelaron como menos importantes el benchmarking (61\%), y de importancia media los casos de estudio y los cursos de formación online, ambas (43\%). 
Figura N.2: Análisis de las actividades formativas

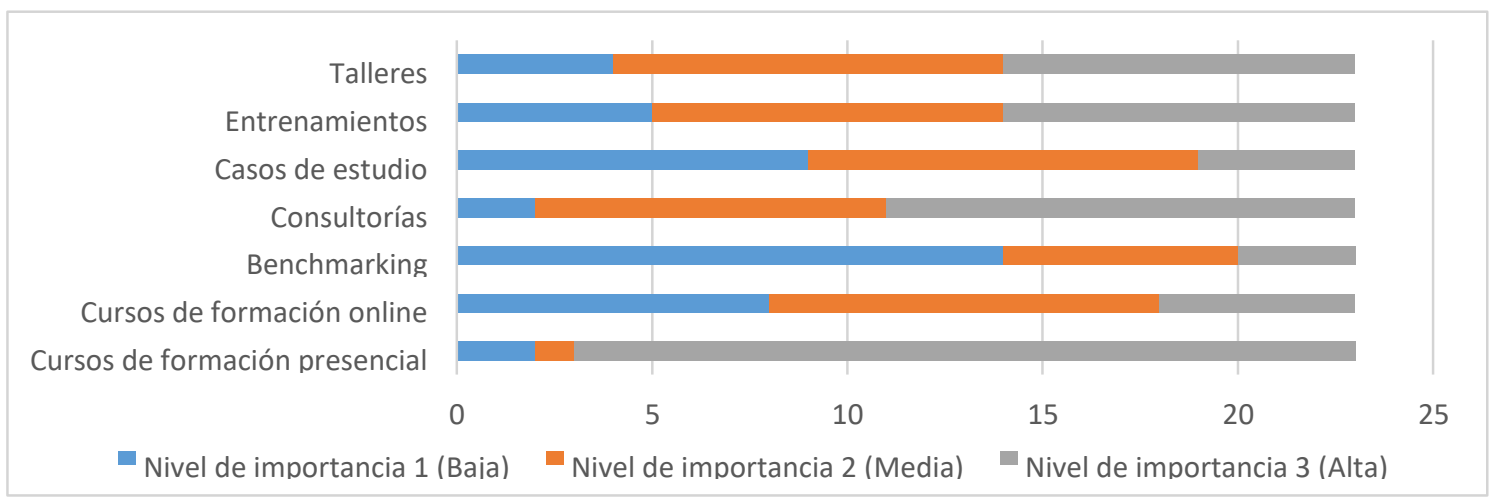

Fuente: Elaboración propia

En el análisis de la encuesta, al brindar a los directivos la oportunidad de exponer las decisiones más significativas que han tenido que enfrentar a lo largo de su experiencia, se detectó como una limitación la disponibilidad del tiempo, ya que la mayoría de los espacios para responder las preguntas de esta índole no fueron completados.

En la facultad estudiada existe un predominio de líderes transformacionales ocupando un total de $70 \%$ en las encuestas, se caracterizan por preocuparse por sus subordinados y compañeros ayudándolos a superar problemas de diferentes índoles, se esfuerzan el doble para lograr las metas del grupo.

Las habilidades que se consideran con alto grado de positividad como se observa en la figura 3 son: la perspectiva estratégica; capacidad para motivar a otros; ayudar al desarrollo de otros; asumir la responsabilidad, perseverancia; compromiso e implicación; honestidad; capacidad de servir a los demás; respeto. Se considera que no están totalmente óptimas: la comunicación; adquisición de experiencia; búsqueda de resultados; innovar y reinventarse; escuchar; persuadir; fortalecer relaciones sociales; delegar trabajo; aptarse al cambio; manejar la crisis; iniciativa y pasión por el trabajo; humildad para reconocer errores propios; detectar oportunidades; organizar; uso de pensamiento crítico y capacidad analizar y resolver.

Figura N.3: Consideraciones generales sobre las habilidades directivas

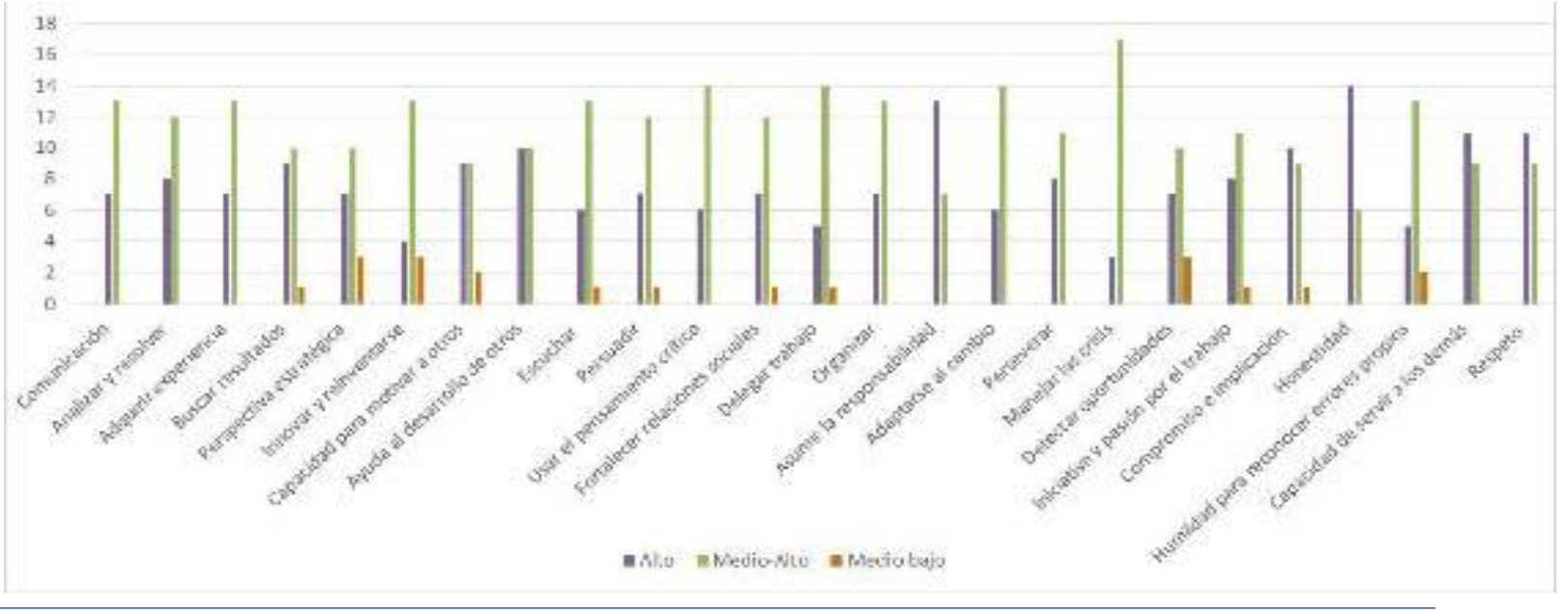


Fuente: Elaboración propia

En la siguiente tabla se establece una relación entre las características más representativas del liderazgo transformacional.

Tabla N.1: Características del liderazgo transformacional

\begin{tabular}{|c|c|c|}
\hline CARACTERISTICAS & CLASIFICACIÓN & HABILIDADES \\
\hline $\begin{array}{c}\text { Se preocupan por sus } \\
\text { subordinados y } \\
\text { compañeros. }\end{array}$ & PERSONAL & $\begin{array}{c}\text { Compromiso e } \\
\text { Implicación }\end{array}$ \\
\hline $\begin{array}{c}\text { Son muy carismáticos y } \\
\text { practican empatía. }\end{array}$ & PERSONAL & Empatía \\
\hline $\begin{array}{c}\text { Logran obtener respeto, } \\
\text { confianza, e inspiran. }\end{array}$ & PERSONAL & Trabajo en Equipo \\
\hline $\begin{array}{c}\text { Ayudan al equipo de } \\
\text { trabajo a superar } \\
\text { problemas de diferentes } \\
\text { índoles. }\end{array}$ & INTERPERSONAL \\
\hline $\begin{array}{c}\text { Hacen partícipe a los } \\
\text { subordinados en el proceso } \\
\text { de toma de decisiones de } \\
\text { la empresa. }\end{array}$ & GESTIÓN & Toma de decisiones \\
\hline
\end{tabular}

Fuente: Elaboración propia

\subsection{La estrategia orientada al desarrollo de habilidades directivas}

La estrategia está dirigida a la transformación del proceso de formación de habilidades en los directivos de la enseñanza superior en turismo. Esta parte del diagnóstico realizado con los directivos de la Facultad de Turismo de la Universidad de La Habana, sobre la situación actual de su proceso de formación, diagnosticando las siguientes regularidades: El proceso no ofrece un programa específico de formación, para la gestión integral de las habilidades, teniendo en cuenta su contexto y sus peculiaridades. En segunda instancia, se observó en el modo de actuación de sus directivos: empirismo, ya que, muchos de ellos trabajan desde la experiencia y no desde la formación profesional especializada.

E1. Determinar las características y el nivel actual de las habilidades directivas personales, interpersonales y grupales.

Conocer el estado actual de las habilidades de los directivos, para contextualizar el proceso de formación, según los diferentes tipos de directivos y el estado de las mismas. 
El objetivo del programa de formación, es capacitar a los cuadros ejecutivos vinculados a la enseñanza superior en turismo de las IES en Cuba, para perfeccionar su modo de actuación, en las tareas de dirección e intervención social, así como la efectividad de su labor.

E2 Programar actividades para la formación de habilidades directivas dentro del sistema de capacitación de los cuadros.

Se propusieron los cursos-talleres de capacitación para los directivos en la UH, iniciando con los vicedecanos de la Facultad de Turismo.

$\checkmark$ Impartir cursos de formación presencial para el desarrollo de habilidades directivas. Coordinar con especialistas las características del programa a desarrollar.

$\checkmark$ Realizar talleres temáticos sobre habilidades y conocimientos entre directivos

$\checkmark$ Crear un espacio que permita el intercambio de experiencias entre los cuadros. Estos cursos-talleres realizados en la UH, seguían el orden siguiente:

- 1ra parte (45 minutos) Conferencia acerca de las habilidades directivas.

- 2da parte (30 minutos) Debate.

- 3ra parte (2 horas) Exposición de la facultad sobre sus acciones estratégicas. Debate.

- 4ta parte (20 minutos) Presentación de temas o programa vinculados a habilidades directivas específicas.

E3: Diseñar un sistema de gestión de la información sobre las habilidades directivas necesarias para el desempeño de los cuadros.

- Impartir conferencias sobre la historia de las habilidades directivas como técnica de gestión y su importancia. Coordinar encuentros entre los directivos de la entidad y especialistas en habilidades directivas como el Dr. Alexis Codina u otros expertos

- Crear un espacio en el mural de la facultad para las habilidades directivas

- Insertar en la página web de la facultad una sección para habilidades directivas

E4: Establecer mecanismos eficientes para desarrollar las habilidades que apoyan el proceso de toma de decisiones y solución de problemas.

$\checkmark$ Utilizar programas informáticos para ejercitar las capacidades de análisis y solución de problemas.

Realizar cursos de creatividad para desarrollar la innovación y la iniciativa. 


\section{Conclusiones}

$>$ El estudio realizado en la Facultad de Turismo de la Universidad de la Habana evidencia que existe un desarrollo positivo de las habilidades directivas en torno al liderazgo, cuyo estilo predominante es el transformacional, como un aspecto favorable que se debe generalizar en todos los directivos.

$>$ Las cuatro estrategias propuestas para mejorar el proceso de desarrollo de habilidades directivas, está fundamentada en una formación específica para superar los problemas actuales y perfeccionar el modo de actuación; una formación definida para los cuadros ejecutivos, que los dotes de los elementos necesarios, para brindar un mejor y más efectivo proceso de formación.

La estrategia involucra la estructuración de acciones de formación, que incluye, el contexto y el sistema de conocimientos propios de la gestión de habilidades directivas para los cuadros de la enseñanza universitaria en turismo, con el fin de ofrecer respuesta a los problemas detectados en el desarrollo de habilidades.

\section{Referencias Bibliográficas}

Astigarraga Gonzalez, E. (2016). Prospectiva Estratégica: orígenes, conceptos claves e introducción a su práctica. Revista Centroamericana de Administración Pública. [en línea] 54-55 : 1-7, 2016. [Consulta: 12 de enero de 2018]. Disponible en: https://biblat.unam.mx / ISBN: 71. 2215-3047 [5]

Bayón, F. (2015). Cómo ha cambiado el concepto de liderazgo: http://www.eoi.es/blogs/fernandobayon/2015/04/24/como-ha-cambiado-elconcepto-de-liderazgo/(22-01-2018) [7].

Chamorro Miranda, D.J. (2015). Factores determinantes del estilo de liderazgo del director-a. Madrid [9].

Codina Jiménez, Alexis. Las habilidades directivas. La Habana Ed. Academia.2016. 120p. ISBN 978-959-237-562-3 [3]

ENPYSC. Estrategia Nacional de Preparación y Superación de los Cuadros del Estado, del Gobierno y sus reservas. Consejo de Estado. Ministerio de Educación Superior. República de Cuba. Editorial Félix Varela. 2010 [1].

Godet, M., ¿Por qué es necesario analizar y construir el futuro? Ed. Donud. 2016[2].

Gómez Ortiz, R. A. (2013). El liderazgo empresarial para la innovación tecnológica en las micro, pequeñas y medianas empresas. [en línea] México. Universidad \& Empresa, vol. 5, núm. 11, diciembre, 62-91[Consulta: 10 de febrero de 2018]. Disponible en: http://www.redalyc.org/ ISSN: 0124-4639 [11]. 
Hatten, K. (1987): Strategic management. Analysis and action, Prentice Hall International, London [10].

Hernández, R; (2014). Metodología de la Investigación. México: Ed.Mc Graw Hill [12].

Ronda Pupo, G.A. Dirección Estratégica, constructo y dimensiones. Ed. Futuro. 2015. 80p. ISBN: 9789592860032 [8].

Whetten, D. A.; Cameron, K. S., Developing Management Skills (eighth edition). Prentice Hall. United States of America. Ed.Perarson 2011.92p.ISBN: ISBN: 9780133127478 [4 y 6]. 


\section{Para citar el artículo indexado.}

Ascón J. \& García M. (2018). Habilidades directivas. Estrategias de formación turística en la Universidad de La Habana. Revista electrónica Explorador Digital 2(1), 45-56. Recuperado desde:

http://cienciadigital.org/revistacienciadigital2/index.php/exploradordigital/article/view/326/7 $\underline{38}$

\section{Ciencia \\ Digital \\ Editorial}

El artículo que se publica es de exclusiva responsabilidad de los autores y no necesariamente reflejan el pensamiento de la Revista Explorador Digital.

El articulo queda en propiedad de la revista y, por tanto, su publicación parcial y/o total en otro medio tiene que ser autorizado por el director o editor de la Revista Explorador Digital.
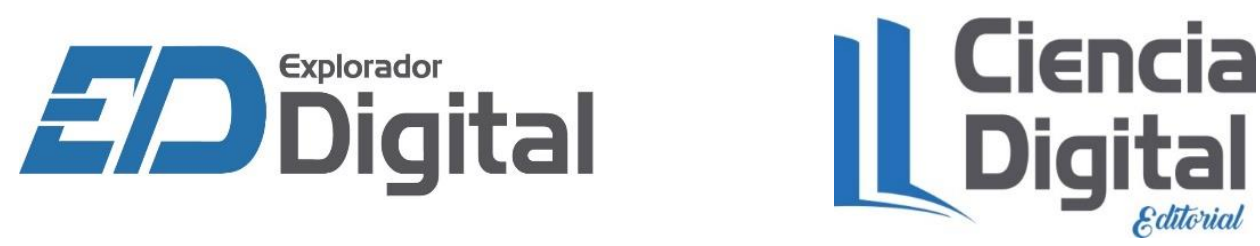